\title{
Hot subdwarfs from the stable Roche lobe overflow channel
}

\author{
S. $\mathrm{Yu}^{1,2}$ and L. $\mathrm{Li}^{2}$ \\ 1 Armagh Observatory, College Hill, Armagh BT61 9DG, Northern Ireland, UK \\ e-mail: syu@arm.ac.uk \\ 2 National Astronomical Observatories Yunnan Observatory, the Chinese Academy of Sciences, PO Box 110, Kunming 650011, \\ PR China
}

Received 25 January 2008 / Accepted 2 June 2009

\section{ABSTRACT}

\begin{abstract}
Context. Hot subdwarfs are core-helium-burning stars with extremely thin envelopes. We discuss the formation and evolution of hot subdwarfs formed through the stable Roche lobe overflow (RLOF) channel of intermediate-mass binaries, although their formation channels are various.

Aims. In this study, we concentrate on the formation and evolution of hot subdwarfs binaries through the stable RLOF channel of intermediate-mass binaries. We aim at setting out the properties of hot subdwarfs and their progenitors, so that we can understand the formation and evolution of hot subdwarfs.

Methods. Employing Eggleton's stellar evolution code, we have computed conservative and nonconservative population I binary evolution sequences. The initial mass of the primary ranges from 2.2 to $6.3 M_{\odot}$, spaced by approximately 0.1 in $\log M$, the initial mass ratio $q_{\mathrm{i}}=M_{1} / M_{2}$ is between 1.1 and 4.5 , and the Roche lobe overflow begins at the main sequence, the Hertzsprung gap and the first giant branch. In nonconservative binary evolution, we assume that 50 percent of the mass lost from the primary leaves the system, carrying away the specific angular momentum of the primary, and the remaining mass is accreted on to the secondary during the RLOF. Also, we have studied the distributions of the mass and orbital periods of hot subdwarfs using the population synthesis approach.

Results. We have obtained the ranges of the initial parameters of progenitor binaries and the properties of hot subdwarfs through the stable RLOF channel of intermediate-mass binaries, e.g. mass, envelope mass and age of hot subdwarfs. We have found that hot subdwarfs could be formed through stable Roche lobe overflow at the main sequence and Hertzsprung gap. We have also found that some subdwarf B or OB stars have anomalously high mass $\left(\sim 1 M_{\odot}\right)$ with a thick envelope $\left(\sim 0.07 M_{\odot}-\sim 0.16\right)$ in our models. By comparing our theoretical results with observations on the hot subdwarfs in open clusters, we suggest that more hot subdwarfs in binary systems might be found in open clusters in the future.
\end{abstract}

Key words. stars: subdwarfs - stars: horizontal-branch - stars: evolution - stars: formation

\section{Introduction}

Hot subdwarfs are generally considered to be core-heliumburning stars with extremely thin hydrogen-rich or heliumrich envelopes. Earlier observations (Humason \& Zwicky 1947; Feige 1958; Greenstein 1966; Slettebak et al. 1961; Klemola 1962; Berger 1963) showed that a number of faint blue field stars with early type at high galactic latitudes display peculiar spectra. Sargent \& Searle (1968) defined subdwarf B (sdB) stars as stars with colors corresponding to those of B stars in which the Balmer lines are abnormally broad compared to population I main sequence B stars and they have weaker HeI lines for their color. Similarly, they defined subdwarf $\mathrm{O}(\mathrm{sdO})$ stars as stars that have strong Balmer lines relative to main sequence stars and in which HeII $\lambda 4686$ is seen. An intermediate class (subdwarf OB, sdOB stars), which has effective temperature and photospheric helium abundances between those of sdB stars and sdO stars, has been reported by Baschek \& Norris (1975) and Hunger et al. (1981). As a blueward extension of the horizontal branch (HB), hot subdwarfs include three subgroups from their spectroscopic classification (Heber 1986):

(i) $\mathrm{sdO}$ - display strong HeII or HeI lines;

(ii) $\mathrm{sdOB}$ - display hydrogen lines and helium lines;

(iii) $\mathrm{sdB}$ - display strong broadended hydrogen lines and weak helium lines.
The origin of hot subdwarfs remains unclear. In the past two decades, two scenarios for the origin of hot subdwarfs have been proposed, i.e. a single star model and a binary model. The single star model was prevalent in the 1990s because of the absence of confirmed hot subdwarfs binaries. The model assumes that the envelope of a giant may be striped off by stellar wind near the tip of the first giant branch (FGB) and leave an almost bare helium core. If the helium core is ignited, the star may become a single hot subdwarf (Lee 1994; Yi et al. 1997; D'Cruz et al. 1996). Sweigart (1997) has studied the evolution of globularcluster stars and suggested that helium mixing driven by internal rotation substantially increases the helium abundance in the envelope. This may result in enhanced mass loss along the FGB and the formation of a hot subdwarf. However, the single star model is inconsistent with the latest observations of hot subdwarfs and the UV upturn of elliptical galaxies which is a strange phenomenon of the rising flux with decreasing wavelength between the Lyman limit and $2500 \AA$ found in almost all elliptical galaxies (Yi \& Yoon 2004; Han et al. 2007). Recent observations indicate that a quantity of hot subdwarfs are found in binaries. Maxted et al. (2001) argued that more than two-thirds of their candidates are binaries with short orbital periods from hours to days, and Napiwotzki et al. (2004) have found that roughly twofifths of their sample are hot subdwarfs binaries. In order to explain the formation of hot subdwarfs in binary, Han et al. (2002) 
suggested a binary model. In such a model, three main channels are responsible for the formation of hot subdwarfs i.e. the common-envelope (CE) ejection channel for hot subdwarf binaries with short orbital periods (0.05-40 days), the stable Roche lobe overflow (RLOF) channel for hot subdwarf binaries with long orbital periods (0.5-2000 days), and the double helium white dwarf (WD) merger channel for single hot subdwarfs. In the $\mathrm{CE}$ ejection channel, the progenitor of a hot subdwarf experiences dynamical mass transfer on the FGB which would result in a $\mathrm{CE}$ and a spiral-in phase, leaving a very close binary after the envelope has been ejected. If the helium core of the giant was ignited, it would become a hot subdwarf in a short-period binary with a companion of a main sequence or white dwarf. In the stable RLOF channel, the progenitor of the hot subdwarf would also lose most of its envelope to produce a hot subdwarf through mass transfer, but the mass transfer would be stable in contrast to the CE ejection channel. In the merger channel, two helium white dwarfs in close binary would be driven together by the orbital angular momentum loss due to gravitational wave radiation or magnetic braking effects. When the white dwarfs merge and the merged object ignites helium, this would produce a single hot subdwarf (Webbink 1984; Iben \& Tutukov 1986; Han 1998). The binary model has explained the observations and the UV upturn of elliptical galaxies (Han et al. 2007).

The majority of field hot subdwarfs found by recent observations (Maxted et al. 2001; Morales-Rueda et al. 2003; Napiwotzki et al. 2004; Edelmann et al. 2005; Morales-Rueda et al. 2005) is in short-period binaries, while few hot subdwarfs in close binaries were found in globular clusters (Moni Bidin et al. 2006a,b). This implies that there would be different formation channels for field and cluster hot subdwarfs. Burleigh et al. (1999) suggested a blue star, which was discovered by Elson et al. (1998) in the young cluster NGC 1818 in the Large Magellanic Cloud (LMC), is a possible hot subdwarf. If hot subdwarfs could exist in very young clusters, their formation would be a puzzle.

Various channels for the formation of hot subdwarfs have been studied in the binary model (Han et al. 2002), as well as the stable RLOF channel by which a low-mass giant loses most of its envelope on the FGB. The giant would leave a degenerate core after the mass transfer stops. If the mass of the degenerate core is high enough, it would experience a helium flash and the star would appear as a core-helium-burning hot subdwarf in a binary. In their model, binary evolution sequences with the donor mass in the range from 0.8 to $1.9 M_{\odot}$ with the accreting components of WD stars or neutron stars (NS) were calculated. In addition, it was assumed that mass transfer takes place at the FGB, and all the mass lost from the system carried away its orbital angular momentum of the accreting component. For the purpose of perfecting the stable RLOF channel and attaining a comprehensive understanding of the formation of hot subdwarfs following Han's calculation, we have executed a computation of evolution sequences of intermediate-mass binaries.

In this study, we have simulated the formation and evolution of hot subdwarfs through the stable RLOF channel in the conservative and nonconservative case. We have taken into account the intermediate mass star filling its Roche lobe at the main sequence (MS), the Hertzsprung gap (HG) or the first giant branch (FGB). The mass transfer stops once the radius of the donor is smaller than its Roche lobe. If the mass of the helium core of the donor (the primary, $M_{1}$ ) is high enough, helium is ignited and the star becomes a hot subdwarf in a binary in which the other component is probably a main sequence star or a subgiant star. We have obtained the properties of the hot subdwarfs and the initial parameters space of their progenitors, e.g. the effective temperature, the surface gravity, mass, envelope mass, lifetime of hot subdwarfs phase. Subsequently, we carried out a Monte Carlo simulation in order to acquire distributions of properties of hot sudwarfs from the stable RLOF channel of intermediate-mass binaries and compared these distributions with observations.

The outline of this paper is as follows. In Sect. 2, we describe the stellar evolution code adopted in this study. In Sect. 3, we present the results. The results are discussed in Sect. 4 and summarized in Sect. 5.

\section{The stellar evolution code and the binary population synthesis code}

\subsection{The stellar evolution code and stellar models}

We use Eggleton's (1971, 1972, 1973) stellar evolution code, which has been updated with the latest physics over the last three decades (Han et al. 1994; Pols et al. 1995, 1998). The code uses a self-adaptive non-Lagrangian mesh and both convective and semiconvective mixing are treated as a diffusion process. The stellar structure equations, the mesh equation and the chemical composition equations are solved simultaneously.

The current code uses an equation of state that includes pressure ionization and Coulomb interaction (Pols et al. 1995), the latest opacity tables derived from Iglesias \& Rogers (1996) and Alexander \& Ferguson (1994a,b) via quadratic interpolation for $X=0.8,0.7,0.5,0.35,0.2,0.1,0$ and for $Y=0.5-Z, 0.2-$ $Z$ and 0 . Nuclear reaction rates come from Caughlan \& Fowler (1988) and Caughlan et al. (1985), and neutrino loss rates are from Itoh et al. (1989, 1992).

We use a typical Population I composition with hydrogen abundance $X=0.700$, helium abundance $Y=0.280$ and metallicity $Z=0.020$ in our computations. We set $\alpha=l / H_{\mathrm{p}}$, the ratio of the mixing length to the local pressure scaleheight is equal to 2 . Such a value of $\alpha$ gives a roughly correct lower main sequence, as determined observationally by Andersen (1991). It also well reproduces the location of the red giant branch in the Hertzsprung-Russell (HR) diagram for stars in the Hyades supercluster (Eggen 1985), as determined by Bessell et al. (1989). A fit to the Sun also result in $\alpha=2$ as the most appropriate choice (Pols et al. 1998).

Convective overshooting is important for the remnant mass of a binary after RLOF evolution because the overshooting directly affects the scale of the nuclear reaction region in a star. In this paper, we follow the work of Schröder et al. (1997) and use an approach based on the stability criterion itself, the $\delta_{\text {ov }}$ prescription, by incorporating a condition that mixing occurs in a region with $\nabla_{r}>\nabla_{a}-\delta$, with $\delta$ defined as the product of a specified constant $\delta_{\mathrm{ov}}$, the overshooting parameter and a conveniently chosen factor that depends only on the ratio $\zeta$ of the radiation pressure to the gas pressure:

$\delta=\delta_{\mathrm{ov}} /\left(2.5+20 \zeta+16 \zeta^{2}\right)$

We take $\delta_{\text {ov }}=0.12$, which leads to overshooting lengths $l_{\text {ov }}$ between 0.25 and $0.32 H_{\mathrm{p}}$ for the mass range of 2.5-7 $M_{\odot}$ with typical $\delta$ values from 0.05 to 0.04 . Such $\delta_{\text {ov }}$ best fits eclipsing binaries (Schröder et al. 1997; Pols et al. 1997), and here we assume that the influence of convective overshooting is the same in our binaries as in the eclipsing binaries. 
RLOF and stellar wind are involved in our models. RLOF is treated as a modification of a surface boundary condition, which is written as:

$\frac{\mathrm{d} m}{\mathrm{~d} t}=C \operatorname{Max}\left[0,\left(\frac{r_{\text {star }}}{r_{\text {lobe }}}\right)^{3}\right]$,

where $\mathrm{d} m / \mathrm{d} t$ is the mass changing rate of the star, $r_{\text {star }}$ is the radius of the star and $r_{\text {lobe }}$ is the radius of its Roche lobe. In principle, the radius of a primary would equal the radius of its Roche lobe if the $C$ is large enough. If the $C$ is very large, however, the Roche lobe overflow would not be continuous. Here we take $C=1000 M_{\odot} \mathrm{yr}^{-1}$ following Han et al. (2002), so that RLOF proceeds steadily and the lobe-filling star overfills its Roche lobe as necessary but never overfills its lobe by much [typically $\left.\left(r_{\text {star }} / r_{\text {lobe }}-1\right) \lesssim 0.001\right]$ (Han et al. 1995, 2000, 2002). For stellar wind, we adopt Reimers' wind (Reimers 1975) massloss law:

$\dot{M}_{\text {wind }}=4 \times 10^{-13} \eta R L / M$,

where we use a coefficient $\eta=1 / 4$ (Carraro et al. 1996; Iben \& Renzini 1983; Renzini 1981).

Our models include two cases for binary evolution:

(a) conservative case;

(b) nonconservative case.

We assume $-\triangle M_{1}$ is lost from the primary, $-\beta \triangle M_{1}$ is accreted on to the secondary, then $-(1-\beta) \triangle M_{1}$ is lost from the system, carrying away the same specific angular momentum as the center of mass of the primary. The change of the angular momentum of the system $\triangle J$ is

$\frac{\triangle J}{J}=\frac{(1-\beta) \triangle M_{1} M_{2}}{M_{1}\left(M_{1}+M_{2}\right)}$,

where $J$ is the orbital angular momentum of the system, $M_{1}$ is the mass of the primary, and $M_{2}$ is the mass of the secondary. We take the mass transfer efficiency $\beta=1.0$ for the conservative case, and $\beta=0.5$ for the nonconservative case, since the results of Paczyński \& Ziółkowski (1967) and Refsdal et al. (1974) indicate that $\beta$ is around 0.5 in the nonconservative case.

The parameter space for the model grid is three dimensional:

(i) the range of initial mass of the primaries $\left(M_{1 \mathrm{i}}\right): \log M_{1 \mathrm{i}}=$ $0.35\left(2.23 M_{\odot}\right) \sim 0.80\left(6.31 M_{\odot}\right)$, and $\triangle \log M_{1 \mathrm{i}}=0.05$;

(ii) the initial mass ratio $q_{\mathrm{i}}=M_{1} / M_{2}: q_{\mathrm{i}}=1.1,1.5,2.0,3.0$, 4.0, 4.2, 4.5;

(iii) the initial orbital periods of the binaries from the minimum period, at which a zero-age main sequence (ZAMS) star would fill its Roche lobe, to the maximum period, at which a star would fill its Roche lobe at the FGB.

In this paper, we focus just on the stable RLOF channel and do not discuss the case of dynamically unstable mass transfer. In each calculation, we checked whether mass transfer was dynamically stable. If the mass transfer rate keeps increasing rapidly, dynamical instability would occur as discussed by Podsiadlowski et al. (2002). This leads to the stellar evolution code breaking down. If mass transfer is stable, we continue mass transfer until the donor shrinks below its Roche lobe, terminating the mass transfer phase. In the case of stable mass transfer, if the mass of the helium core exceeds the appropriate minimum core mass for helium ignition, it will appear as a core-helium-burning hot subdwarf.

\subsection{Monte Carlo simulation parameters}

In order to obtain distributions of properties of hot subdwarfs from the stable RLOF channel of intermediate-mass binaries, we have performed a simple Monte Carlo simulation where we follow the evolution of a sample of a million binaries. The physical inputs of the simulation are depicted as follows:

(i) we assume that the star formation rate (SFR) is constant over the last $13.5 \mathrm{Gyr}$;

(ii) for the initial mass function (IMF) of the primary, we adopted the IMF of Miller \& Scalo (1979); the primary mass is generated using the formula of Eggleton et al. (1989),

$$
M_{1}=\frac{0.19 X}{(1-X)^{0.75}+0.032(1-X)^{1 / 4}},
$$

where $X$ is a random number uniformly distributed between 0 and 1 . The studies by Kroupa et al. (1993) and Zoccali et al. (2000) support this IMF;

(iii) for the initial mass ratio distribution, we suppose a constant one (Mazeh et al. 1992; Goldberg \& Mazeh 1994),

$n(1 / q)=1,0 \leqslant 1 / q \leqslant 1$,

where $q_{\mathrm{i}}=M_{1} / M_{2}$;

(iv) for the distribution of initial orbital separations, we employed that used by Han et al. (2003), where they assume that all stars are members of binary systems and that the distribution of separations is constant in $\log a$ ( $a$ is the separation) for wide binaries and falls off smoothly at close separations:

$\operatorname{an}(a)=\left\{\begin{array}{l}\alpha_{\mathrm{sep}}\left(\frac{a}{a_{0}}\right)^{m}, a \leqslant a_{0}, \\ \alpha_{\mathrm{sep}}, a_{0}<a<a_{1} .\end{array}\right.$

where $\alpha_{\text {sep }} \approx 0.070, a_{0}=10 R_{\odot}, a_{1}=5.75 \times 10^{6} R_{\odot}=$ $0.13 \mathrm{pc}, m \approx 1.2$. This distribution implies that there is an equal number of wide binary systems per logarithmic interval, and that approximately 50 per cent of stellar systems are binary systems with orbital periods less than $100 \mathrm{yr}$.

The total numbers of observed hot subdwarfs are associated with their evolution lifetime. Each binary system in our models is multiplied by a factor of $\gamma_{\mathrm{t}}$, where $\gamma_{\mathrm{t}}=t / 2 \times 10^{8} \mathrm{yrs}, t$ is the lifetime of hot subdwarf phase.

\section{Results}

In general, three subgroups are defined from photometric and spectroscopic classifications: $\mathrm{sdB}, \mathrm{sdOB}$ and sdO. Figure 1 displays the position of observed hot subdwarfs. It is seen in Fig. 1 that there is a boundary between $\mathrm{sdB}, \mathrm{sdOB}$ and $\mathrm{sdO}$ stars at effective temperature $T_{\text {eff }} \approx 35000 \mathrm{~K}$. The effective temperature of sdB and sdOB stars is between $20000 \mathrm{~K}$ and $35000 \mathrm{~K}$ while that of sdO is between $35000 \mathrm{~K}$ and $80000 \mathrm{~K}$. Due to the tight correlation of spectral type (effective temperature, $T_{\text {eff }}$ ) and spectroscopy, we define three subgroups from spectral type $\left(T_{\text {eff }}\right)$ classification corresponding to the spectroscopic classification:

(1) $\mathrm{sdB}-T_{\text {eff }}$ is between $20000 \mathrm{~K} \sim 35000 \mathrm{~K}$;

(2) $\mathrm{sdOB}-T_{\text {eff }}$ is between $35000 \mathrm{~K} \sim 40000 \mathrm{~K}$;

(3) $\mathrm{sdO}-T_{\text {eff }}$ is between $40000 \mathrm{~K} \sim 80000 \mathrm{~K}$.

All of them have helium-burning-cores, and their logarithmic surface gravities $(\log (g))$ are between 4.5 and $6.5(\mathrm{cgs})$. We distinguish $\mathrm{sdB}$ and $\mathrm{sdOB}$ stars by effective temperature for discussion, although their differences are also associated with surface 


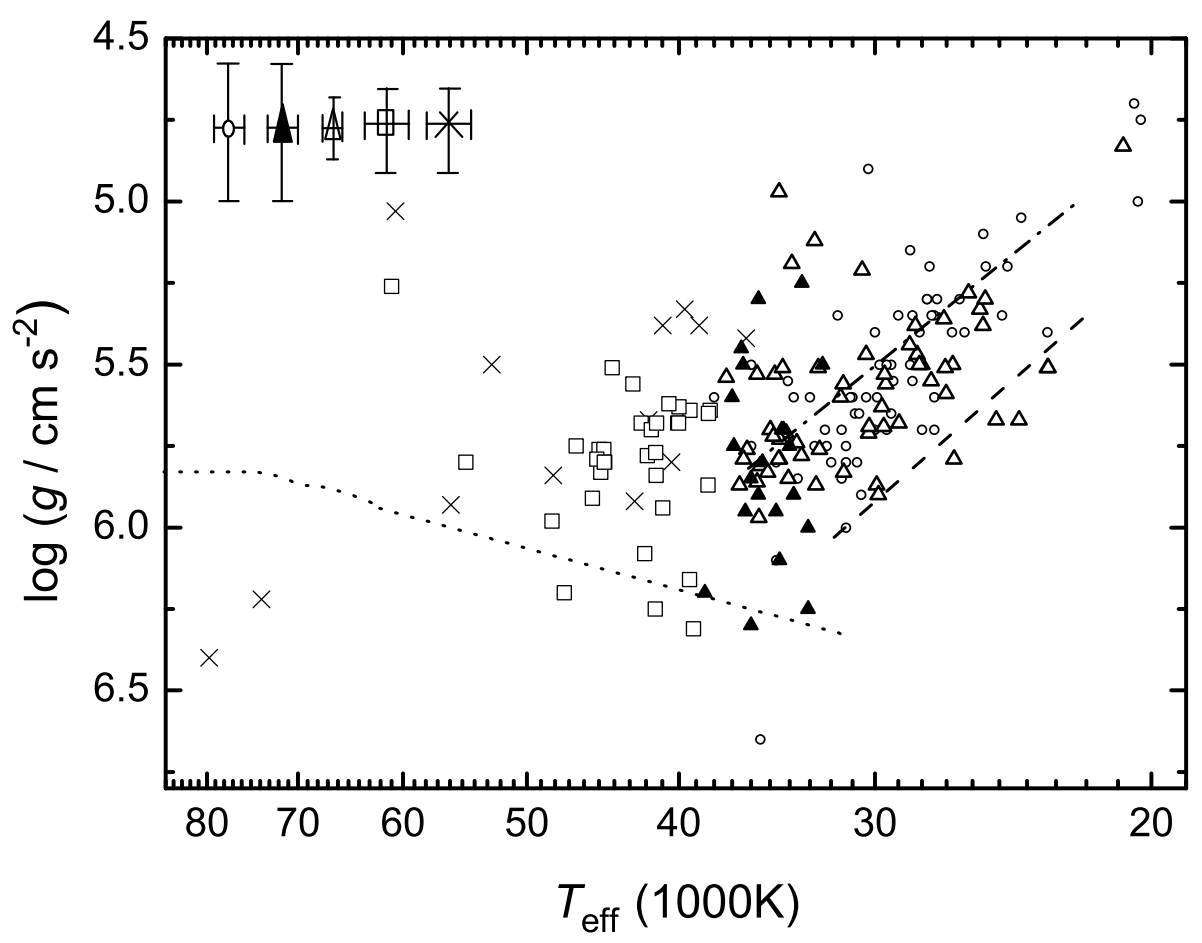

Fig. 1. Logarithmic surface gravities versus effective temperature of observed hot subdwarfs (see typical error-bars in the upper left side). Open circles and solid triangles are for $\mathrm{sdB}$ and sdOB stars observed by Edelmann et al. (2003). Open triangles are for sdB stars observed by Lisker et al. (2005). Crosses are for sdO stars observed by Stroeer et al. (2007). Dashed line, dot-dashed line and dotted line indicate ZAEHB (the Zero-Age Extreme Horizontal Branch, assuming the mass of hot subdwarf is $0.5 M_{\odot}$ ), TAEHB (the Terminal-Age Extreme Horizontal Branch, assuming the mass of hot subdwarf is $0.5 M_{\odot}$ ) (Dorman et al. 1993) and He-ZAMS (the Helium Zero-Age Main Sequence) (Paczyński 1971).

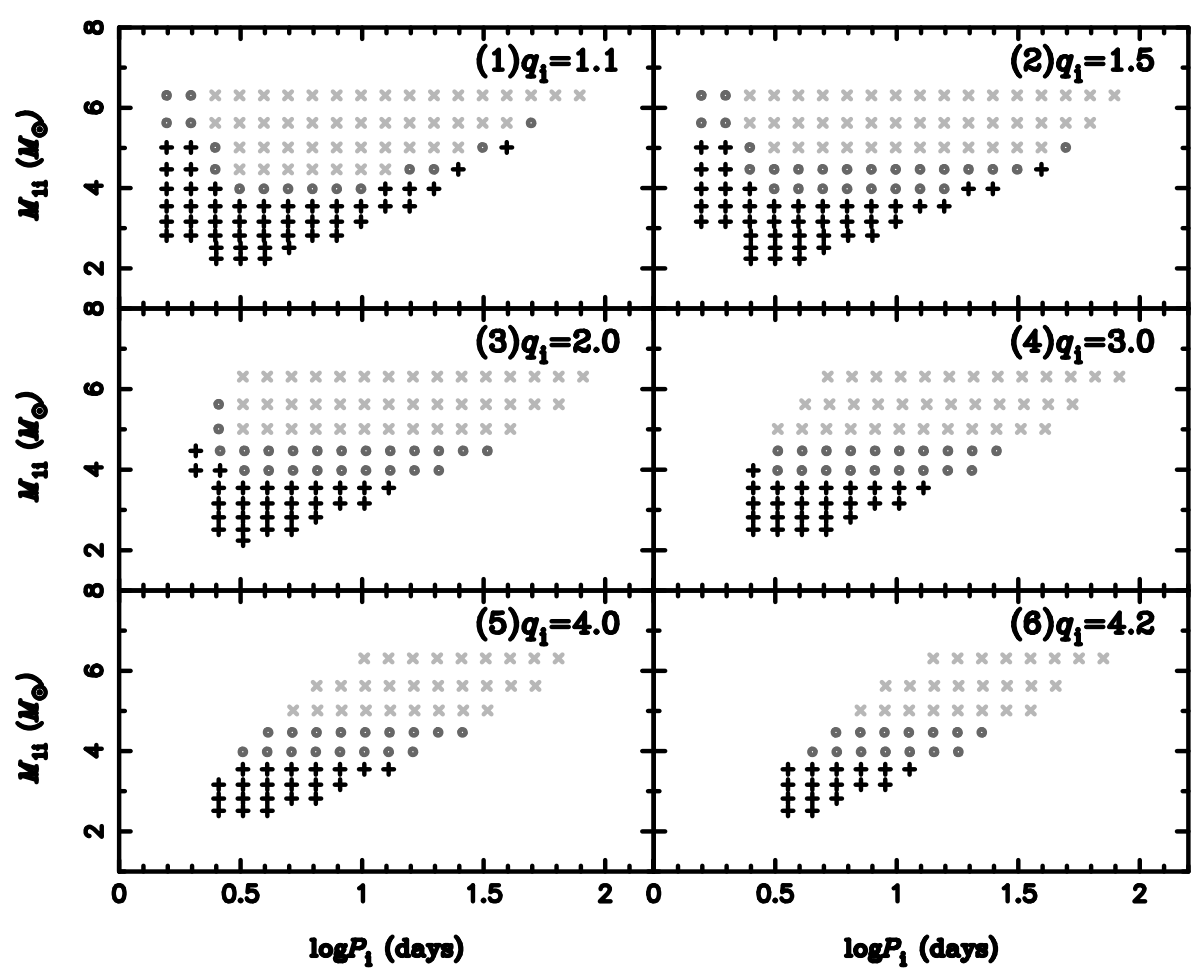

Fig. 2. Initial parameters of progenitors of hot subdwarfs in the conservative case. Pluses (black) are for progenitors of sdB stars, circles (dark grey) for progenitors of sdOB stars, and crosses (light grey) for progenitors of sdO stars. Panels (1)-(6) indicate the mass ratio $q_{\mathrm{i}}$ of 1.1, $1.5,2.0,3.0,4.0,4.2$, respectively. helium abundance. In fact, $\mathrm{sdOB}$ and $\mathrm{sdB}$ stars are mixed in the region $34000-38000 \mathrm{~K}$ in the $T_{\text {eff }}-\log (g)$ diagram.

\subsection{The initial parameter space}

We are interested in the ranges of the initial parameters of the progenitors of hot subdwarfs through the stable RLOF channel of intermediate-mass binaries, i.e. the primary mass $\left(M_{\mathrm{i}}\right)$, the mass ratio $\left(q_{\mathrm{i}}\right)$, the orbital periods $\left(\log P_{\mathrm{i}}\right)$. Figures 2 and 3 show the initial parameter space of the progenitors of hot subdwarfs in conservative case and nonconservative case.
From Figs. 2 and 3, we see that the number of binary systems experiencing stable mass transfer decreases with increasing mass ratio $q_{\mathrm{i}}$. When $q_{\mathrm{i}}>3.0$, a large number of binary systems in the conservative case have mass transfer rates higher than $10^{-5} M_{\odot} \mathrm{yr}^{-1}$ (the light grey region). The number of binaries with stable mass transfer in the nonconservative case is more than that in the conservative case due to mass loss from binaries. The primary lying in the light grey region in Figs. 2 and 3 would become a hot subdwarf in $\sim 10^{6}$ yrs after it experiences a stable mass transfer with a rate higher than $10^{-5} M_{\odot} \mathrm{yr}^{-1}$ which last $\sim 10^{5}$ to $\sim 10^{6} \mathrm{yrs}$. Given $q_{\mathrm{i}}>4.2$ in the conservative case $\left(q_{\mathrm{i}}>4.5\right.$ in the nonconservative case), the mass transfer of all 


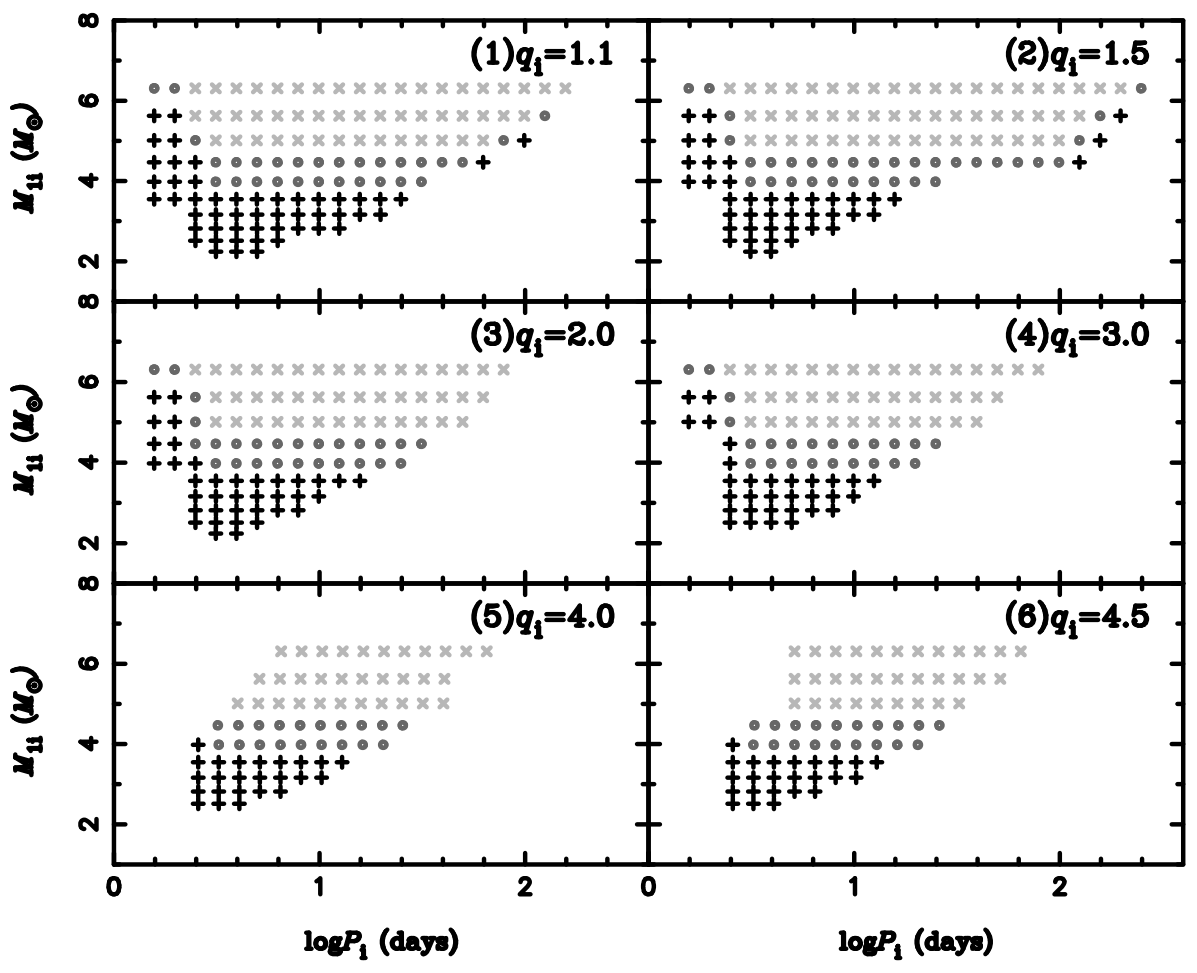

Fig. 3. Initial parameters of progenitors of hot subdwarfs in the nonconservative case. Pluses (black) are for progenitors of sdB stars, circles (dark grey) for progenitors of sdOB stars, and crosses (light grey) for progenitors of sdO stars. Panels (1)-(6) indicate the mass ratio $q_{\mathrm{i}}$ of 1.1 , $1.5,2.0,3.0,4.0,4.5$, respectively. systems in our models is dynamically unstable, and the stellar evolution code breaks down.

The formation of different types of hot subdwarfs depends greatly on the initial parameters (i.e. initial mass, initial orbital periods) of binaries, although this relationship becomes weaker if $q_{\mathrm{i}} \geq 4$.0. We depict these relationships as follows:

(i) we can understand the relationship between the formation of different types of hot subdwarfs and the initial mass of their progenitors. A star with a larger initial mass could leave a larger helium core. If the helium core is ignited, the star becomes a hot subdwarf with an earlier spectral type;

(ii) the relationship between the formation of different types of hot subdwarfs and the initial orbital periods is more complicated than the relationship mentioned above.

From Figs. 2 and 3, for a given initial mass, such as $M_{\mathrm{i}}=$ $5.01 M_{\odot}$, we can see that, if $q_{\mathrm{i}} \leq 3.0$, it may produce sdB stars (sdOB or sdO stars) when the initial orbital periods are shorter than 2.1 days (longer than 2.1 days); if $q_{\mathrm{i}}>3.0$, only sdO stars will be produced. This is because an MS star fills its Roche lobe and this results in the onset of the mass transfer at the MS stage. In this case, a star loses more mass because of a longer mass transfer time, so that a smaller helium core and a thinner envelope would be left, when the star enters the extreme horizontal branch (EHB) stage.

In addition, if $q_{\mathrm{i}} \leq 1.5$, for a given initial mass, such as $M_{\mathrm{i}}=5.01 M_{\odot}$, we can see that $\mathrm{sdB}$ or sdOB stars (sdO stars) may also be produced when the initial orbital period is longer than 31.6 days (shorter than 31.6 days but longer than 2.1 days), where the mass transfer begins at the FGB stage. In this case, the timescale of the mass transfer is very short, as the primary contracts soon. This leads to less mass lost from the primary, leaving an sdB star with a larger helium core and thicker envelope.

\subsection{The $T_{\text {eff }}-\log (g)$ diagram}

The evolution tracks of some hot subdwarfs with different initial parameters are shown in Fig. 4. Panels (a) and (b) are for the conservative case while Panels (c) and (d) for the nonconservative case.

As seen from Fig. 4, the effective temperatures $T_{\text {eff }}$ of hot subdwarfs increase with increasing initial mass while their logarithmic surface gravities $\log (g)$ decrease. This is because a star with higher initial mass could more easily leave a larger helium core. For a given initial mass, $\log (g)$ of hot subdwarfs decreases with increasing initial orbital periods.

We find that the relationship between $T_{\text {eff }}$ and $P_{\mathrm{i}}$ is somewhat strange both in the conservative and nonconservative case, especially for given $q_{\mathrm{i}} \leq 1.5$ and $M_{\mathrm{i}} \geq 4.0 M_{\odot}$. As mentioned in Sect. 3.1, this phenomenon is associated with the mass of the envelope of hot subdwarfs. In order to display the relationship between $T_{\text {eff }}$ and $P_{\mathrm{i}}$ clearly, we plot in Fig. 5 the evolution tracks of hot subdwarfs with different initial mass and orbital periods in the $T_{\text {eff }}-\log (g)$ diagram when $q_{\mathrm{i}}=1.5$. For a given initial mass, such as $5.01 M_{\odot}, T_{\text {eff }}$ would increase and then it would decline with increasing $P_{\mathrm{i}}$.

From Fig. 4, we can see that there is a lack of observed hot subdwarfs in the upper left region of the $T_{\text {eff }}-\log (g)$ diagram. This is consistent with our models (Note that the evolution curves in Fig. 4 are at the boundary for producing hot subdwarfs.). In addition, if the mass of the helium core of a star is too small to be ignited, it will not experience the core-heliumburning stage. So, it is difficult to observe hot subdwarfs in the lower right region of the $T_{\text {eff }} \log (g)$ diagram.

The evolutionary tracks of hot subdwarfs in our models can cover positions of observed hot subdwarfs except for a few observed hot subdwarfs with effective temperatures between $39000 \mathrm{~K}$ and $50000 \mathrm{~K}$ and logarithmic surface gravities between 6.1 and 6.4 (cgs). The origin of these hot subdwarfs is not clear. 

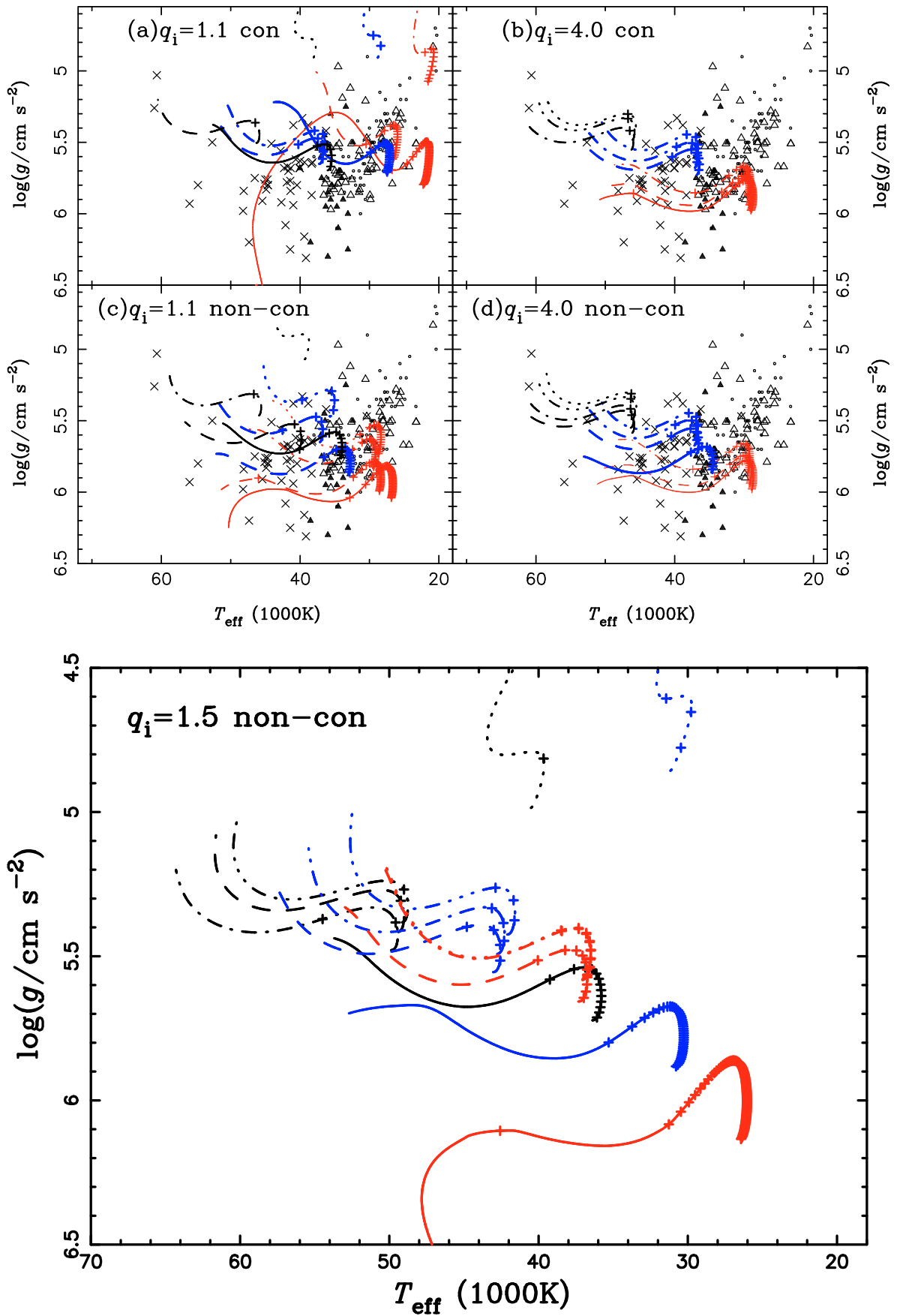

Fig. 4. Evolutionary tracks of hot subdwarfs with different initial mass ratios in the $T_{\text {eff- }}-\log g$ diagram. Panels a) $(q=1.1)$ and $\mathbf{b})(q=$ 4.0) are for the conservative case. Panels c) $(q=1.1)$ and d) $(q=4.0)$ are for the nonconservative case. Circles and triangles are for $\mathrm{sdB}$ stars from the Hamburg quasar survey (Edelmann et al. 2003) and the ESO supernova Ia progenitor survey (Lisker et al. 2005), respectively. Squares are for sdO stars from the ESO supernova Ia progenitor survey (Stroeer et al. 2007). Red curves, blue curves and black curves are for the initial primary mass of $2.82 M_{\odot}, 3.98 M_{\odot}$ and $5.62 M_{\odot}$. In panel a), solid curves are for initial orbital periods of 1.6 days, dashed for 5.0 days, dot-dashed for 7.9 days, blue dotted curves for 20.0 days, and black dotted curves for 31.6 days. In panel $\mathbf{b}$ ), solid curves are for initial orbital periods of 2.6 days, dashed for 3.2 days, dot-dashed for 6.5 days, dash-dot-dot-dot for 20.0 days, dotted for 50.1 days. In panel c), solid curves are for initial orbital periods of 1.6 days, dashed for 2.5 days, dot-dashed for 6.5 days, red dotted for 12.6 days, blue dotted for 31.5 days, and black dotted for 48.5 days. In panel d), solid curves are for initial orbital periods of 2.5 days, red and blue dashed for 3.2 days, black dashed for 5.1 days, dot-dashed for 8.1 days, dash-dotdot-dot for 20.4 days, dotted for 32.4 days. The age differences between adjacent crosses are $10^{7} \mathrm{yrs}$.
Fig. 5. Evolutionary tracks of hot subdwarfs with mass ratio $q=1.5$ in the $T_{\text {eff }}-\log g$ diagram for the nonconservative case. Red curves, blue curves and black curves are for the initial primary mass of $3.98 M_{\odot}, 5.01 M_{\odot}$ and $6.31 M_{\odot}$. Solid curves are for initial orbital periods of 1.6 days, dashed for 6.3 days, dot-dashed for 24.9 days, dash-dotdot-dot for 99.1 days, blue dotted for 157.0 days and black dotted for 248.9 days. The age differences between adjacent crosses are $5.0 \times 10^{6}$ yrs.

\subsection{The mass, the envelope mass and the orbital periods of hot subdwarfs}

We have obtained the mass and the envelope mass of hot subdwarfs which depend strongly on the initial parameters of their progenitors, i.e. the primary mass $\left(M_{1 \mathrm{i}}\right)$, the orbital periods $\left(P_{\mathrm{i}}\right)$ and the mass ratio $\left(q_{\mathrm{i}}\right)$. The mass and the envelope mass of hot subdwarfs for the conservative case are shown in Figs. 6 and 7, and they are shown in Figs. 8 and 9 for the nonconservative case.
The relationship between the envelope mass of hot subdwarfs and the initial parameters of their progenitors can help us to understand the post-evolution of hot subdwarfs. As shown by Figs. 7 and 9, the envelope mass has a weak dependence on the initial mass ratio if $q_{\mathrm{i}} \geq 2.0$ while the envelope mass has a clearly wider range if $q_{\mathrm{i}} \leq 1.5$. For a given initial mass and initial mass ratio, the longer the initial orbital periods, the higher the envelope mass. Hot subdwarfs would enter asymptotic giant branch manqué (AGB-manqué) stage after their core helium exhaustion if their envelope masses are between $\sim 0.01$ and $0.04 M_{\odot}$; hot 

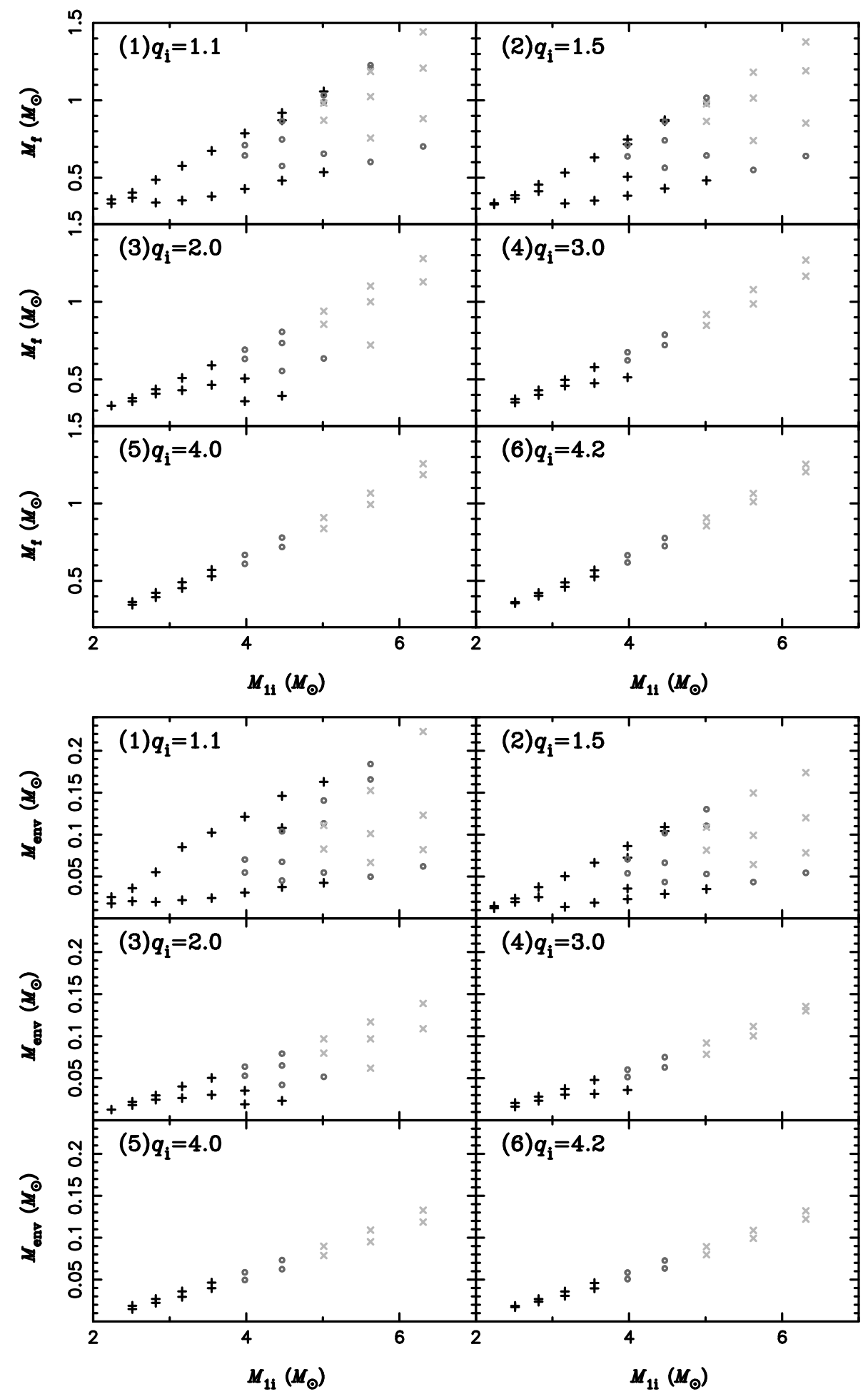

Fig. 6. The mass of hot subdwarfs $\left(M_{\mathrm{f}}\right)$ versus the mass of their pogenitors $\left(M_{1 \mathrm{i}}\right)$ in the conservative case. Pluses (black) are for sdB stars, circles (dark grey) for sdOB stars, and crosses (light grey) for sdO stars. Panels (1)-(6) indicate the mass ratio $q_{\mathrm{i}}$ of $1.1,1.5,2.0,3.0,4.0$, 4.2 , respectively.
Fig. 7. The envelope mass of hot subdwarfs $\left(M_{\text {env }}\right)$ versus the mass of their pogenitors $\left(M_{1 \mathrm{i}}\right)$ in the conservative case. Pluses (black) are for sdB stars, circles (dark grey) for sdOB stars, and crosses (light grey) for sdO stars. Panels (1)-(6) indicate the mass ratio $q_{\mathrm{i}}$ of 1.1 , $1.5,2.0,3.0,4.0,4.2$, respectively. subdwarfs with an envelope mass of $\sim 0.04 \sim 0.08 M_{\odot}$ would undergo the early asymptotic giant branch (early-AGB) stage; hot subdwarfs with the envelope mass larger than $\sim 0.08 M_{\odot}$ would become normal asymptotic giant branch (AGB) stars (see Fig. 10 for an example).

As seen from Figs. 6-9, a few sdB stars have progenitors with a mass of 3.98-5.01 $M_{\odot}$ if $q_{\mathrm{i}} \leq 3.0$, while the majority of $\mathrm{sdB}$ stars come from stars with mass of $2.23-3.55 M_{\odot}$. This is because a primary with a high initial mass would lose more mass after a long mass transfer phase due to the primary filling its Roche lobe at the MS stage. The progenitors of sdO (sdOB) stars have high mass in general, e.g. $\geq 5.01 M_{\odot}\left(3.98 M_{\odot}-4.47 M_{\odot}\right)$.
In particular, we find that if the mass ratio $q_{\mathrm{i}} \leq 1.5$, there are some $\mathrm{sdB}$ or $\mathrm{sdOB}$ stars with anomalously high mass and envelope mass ("anomalous" sdB or sdOB stars, see Table 2) formed though the stable RLOF channel, whose progenitors have a high initial mass and a long initial orbital period, such as $M_{1 \mathrm{i}}=5.01 M_{\odot}, P_{\mathrm{i}}=40.6$ days (see Table 3 ). These results are consistent with the initial-final mass relation discussed by Han et al. (2000), Chen et al. (2002, 2003).

In order to obtain the mass distribution of hot subdwarfs and the final orbital period distribution of the binaries in this work, we have plotted Figs. 11 and 12 by executing a simple Monte Carlo simulation as mentioned in Sect. 2.2. It is seen that the 

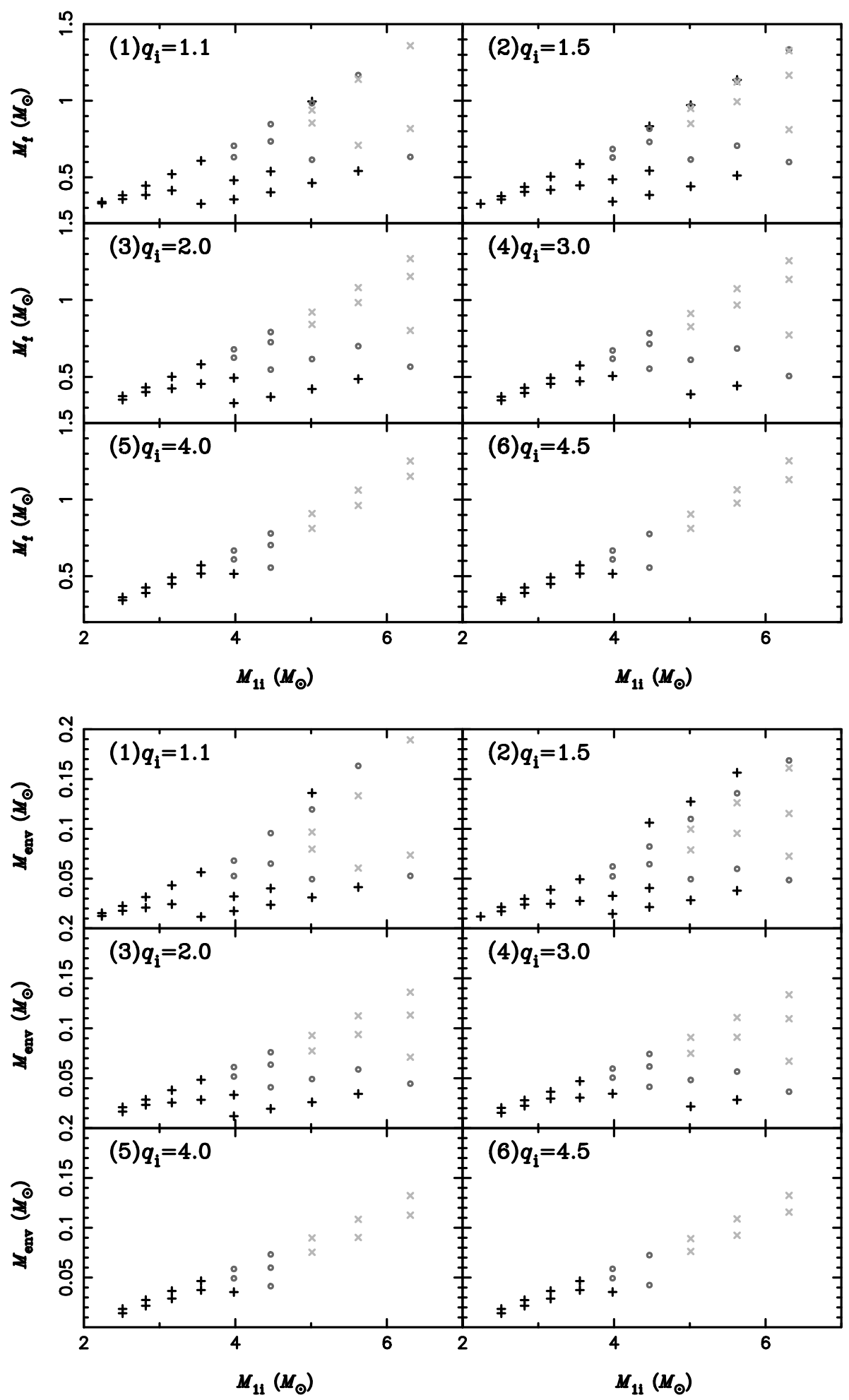

Fig. 8. The mass of hot subdwarfs $\left(M_{\mathrm{f}}\right)$ versus the mass of their pogenitors $\left(M_{1 \mathrm{i}}\right)$ in the nonconservative case. Pluses (black) are for $\mathrm{sdB}$ stars, circles (dark grey) for sdOB stars, and crosses (light grey) for sdO stars. Panels (1)-(6) indicate the mass ratio $q_{\mathrm{i}}$ of $1.1,1.5,2.0,3.0$, $4.0,4.5$, respectively.
Fig. 9. The envelope mass of hot subdwarfs $\left(M_{\text {env }}\right)$ versus the mass of their pogenitors $\left(M_{1 \mathrm{i}}\right)$ in the nonconservative case. Pluses (black) are for sdB stars, circles (dark grey) for sdOB stars, and crosses (light grey) for sdO stars. Panels (1)-(6) indicate the mass ratio $q_{\mathrm{i}}$ of 1.1 , $1.5,2.0,3.0,4.0,4.5$, respectively. mass of hot subdwarfs are in a wide range from 0.33 to $1.22 M_{\odot}$ with a peak near $0.45 M_{\odot}$. The orbital periods of the binaries are also in a wide range from 5.0 to 900.0 days, and peak at around 120.0 days in the conservative case while the peak at around 30.0 days in the nonconservative case.

In Fig. 12, we have also plotted the orbital periods of observed binary systems containing hot subdwarfs (Morales-Rueda et al. 2003; Edelmann et al. 2005; Napiwotzki et al. 2004) as short bars with different colors. A few observed binaries with long orbital periods could be formed through the stable RLOF channel while the majority of observed binaries with short orbital periods probably would come from the CE ejection channel (Han et al. 2002).

\subsection{The onset of mass transfer at different stage}

The properties of hot subdwarfs are significantly affected by the stage where mass transfer began in their progenitors. When the onset of mass transfer is at the main sequence, the primary would lose an amount of mass. If its helium core is ignited, the primary would become a hot subdwarf with a low mass and a low envelope mass. Table 3 lists the parameters of hot subdwarfs and their 


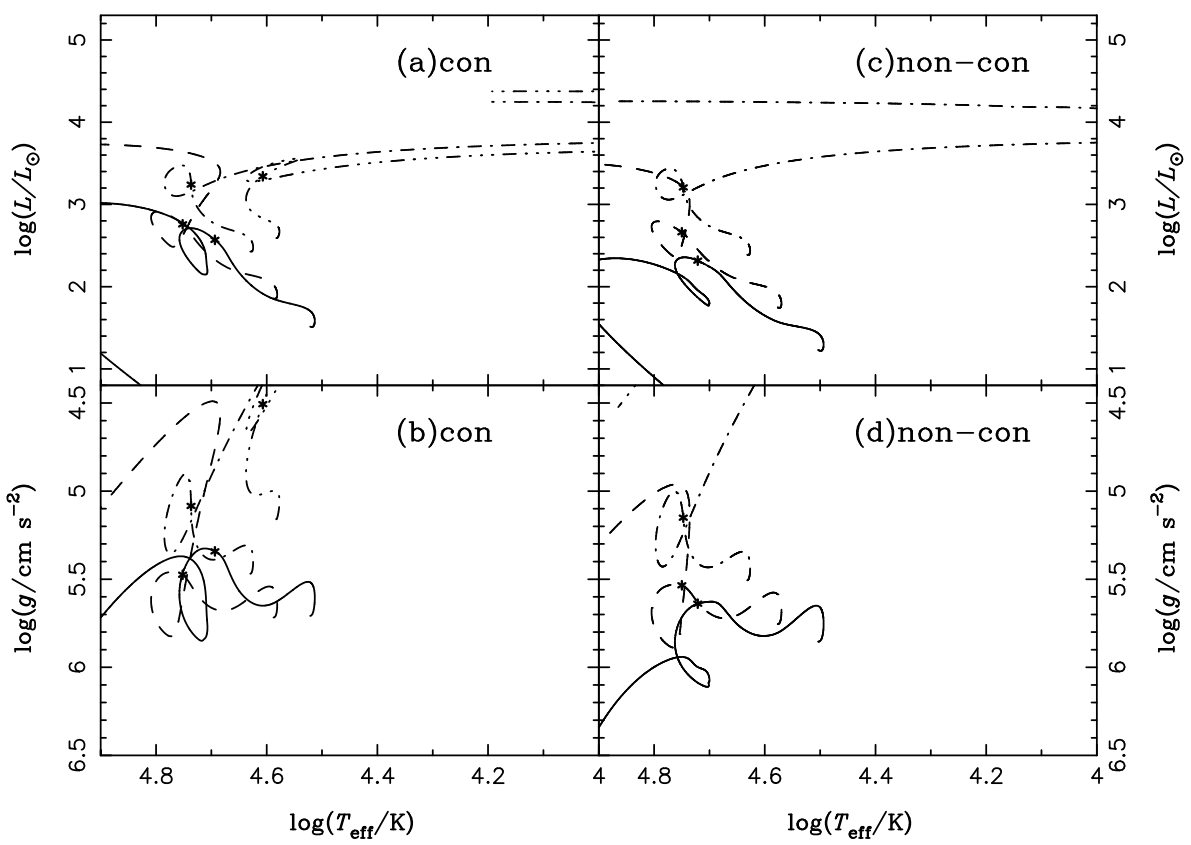

Fig. 10. The HR diagrams and the $T_{\text {eff }}-\log (g)$ diagrams of hot subdwarfs where the initial mass $\left(M_{1 \mathrm{i}}\right)$ is $5.01 M_{\odot}$ and the mass ratio $q_{\mathrm{i}}$ is 1.1. Solid curves are for initial orbital periods of 1.6 days ( $\mathrm{sdB}$ stars with envelope mass of $0.04 M_{\odot}$ in left panel, envelope mass of $0.03 M_{\odot}$ in right panel), dashed for 2.5 days (sdOB stars with envelope mass of $0.05 M_{\odot}$ ), dot-dashed for 15.7 days (sdO stars with envelope mass of $0.09 M_{\odot}$ ), dash-dot-dot-dot for 31.3 days (left panel, sdOB star with envelope mass of $0.12 M_{\odot}$ ). Panels a) and $\mathbf{b}$ ) are for the conservative case. Panels c) and d) are for the nonconservative case. Asterisks indicate that helium is exhausted in its center.
Table 1. The mass and the envelope mass of normal hot subdwarf stars.

\begin{tabular}{lcc}
\hline \hline Type & $\begin{array}{c}M_{\mathrm{f}} \\
\left(M_{\odot}\right)\end{array}$ & $\begin{array}{c}M_{\text {env }} \\
\left(M_{\odot}\right)\end{array}$ \\
\hline conservative case: & & \\
sdB & $0.33-0.67$ & $0.01-0.05$ \\
sdOB & $0.61-0.86$ & $0.05-0.10$ \\
sdO & $0.75-1.44$ & $0.07-0.22$ \\
\hline nonconservative case: & & \\
sdB & $0.32-0.61$ & $0.01-0.05$ \\
sdOB & $0.60-0.85$ & $0.05-0.09$ \\
sdO & $0.81-1.36$ & $0.08-0.19$ \\
\hline
\end{tabular}

$M_{\mathrm{f}}=$ the mass of hot subdwarfs; $M_{\text {env }}=$ the envelope mass of hot subdwarfs.

Table 2. The mass and the envelope mass of anomalous hot subdwarfs for mass ratios $q_{\mathrm{i}}=1.1,1.5$.

\begin{tabular}{lcc}
\hline \hline Type & $\begin{array}{c}M_{\mathrm{f}} \\
\left(M_{\odot}\right)\end{array}$ & $\begin{array}{c}M_{\text {env }} \\
\left(M_{\odot}\right)\end{array}$ \\
\hline conservative case: & & \\
sdB & $0.72-1.06$ & $0.07-0.16$ \\
sdOB & $0.98-1.23$ & $0.11-0.19$ \\
\hline nonconservative case: & & \\
sdB & $0.83-1.13$ & $0.10-0.16$ \\
sdOB & $0.97-1.17$ & $0.11-0.16$ \\
\hline
\end{tabular}

$M_{\mathrm{f}}=$ the mass of hot subdwarfs; $M_{\mathrm{env}}=$ the envelope mass of hot subdwarfs.

progenitors where mass transfer begins at the main sequence, Hertzsprung gap or first giant branch in the conservative case and the nonconservative case.

From our calculations, we found that the properties of the hot subdwarfs strongly depend on the initial orbital period when $q_{\mathrm{i}} \leq 1.5$, but the dependence becomes weaker for hot subdwarfs with high mass ratios. Figures 10 and 13 show the HertzsprungRussell (HR) diagrams and the $T_{\text {eff }}-\log (g)$ diagrams of hot subdwarfs for a given initial primary mass with a different initial mass ratio $\left(q_{\mathrm{i}}=1.1\right.$ and 4.0$)$ when mass transfer begins at different stages in the conservative and nonconservative cases. From Figs. 10 and 13, we can see that the onset of mass transfer at
Table 3. Parameters of some typical hot subdwarfs and their progenitors.

\begin{tabular}{lcccc}
\hline \hline $\begin{array}{l}M_{1 \mathrm{i}} / M_{\mathrm{f}}(\mathrm{type}) \\
\left(M_{\odot}\right)\end{array}$ & $q_{\mathrm{i}}$ & $\begin{array}{c}P_{\mathrm{i}} / P_{\mathrm{f}} \\
(\text { days })\end{array}$ & $\begin{array}{c}M_{\text {env }} \\
\left(M_{\odot}\right)\end{array}$ & $\begin{array}{c}t_{\text {age }} \\
(\mathrm{yrs})\end{array}$ \\
\hline conservative case: & & & & \\
$3.55 / 0.38(\mathrm{sdB})$ & 1.1 & $1.5 / 151.1$ & 0.02 & $3.8 \times 10^{8}$ \\
$3.55 / 0.56(\mathrm{sdB})$ & 1.1 & $3.5 / 116.1$ & 0.04 & $0.9 \times 10^{8}$ \\
$3.55 / 0.53(\mathrm{sdB})$ & 4.0 & $3.5 / 11.5$ & 0.04 & $1.1 \times 10^{8}$ \\
$5.01 / 0.54(\mathrm{sdB})$ & 1.1 & $1.6 / 147.8$ & 0.04 & $1.0 \times 10^{8}$ \\
$5.01 / 0.65(\mathrm{sdOB})$ & 1.1 & $2.5 / 137.3$ & 0.05 & $0.6 \times 10^{8}$ \\
$5.01 / 0.93(\mathrm{sdO})$ & 1.1 & $15.7 / 317.2$ & 0.09 & $0.2 \times 10^{8}$ \\
$5.01 / 1.01(\mathrm{sdOB})$ & 1.1 & $31.3 / 483.8$ & 0.12 & $0.2 \times 10^{8}$ \\
$5.01 / 1.06(\mathrm{sdB})$ & 1.1 & $40.6 / 545.5$ & 0.16 & $0.1 \times 10^{8}$ \\
$5.01 / 0.84(\mathrm{sdO})$ & 4.0 & $5.1 / 13.1$ & 0.08 & $0.2 \times 10^{8}$ \\
$5.01 / 0.91(\mathrm{sdO})$ & 4.0 & $32.3 / 60.8$ & 0.09 & $0.2 \times 10^{8}$ \\
\hline non-conservative case: & & & & \\
$3.55 / 0.33(\mathrm{sdB})$ & 1.1 & $1.5 / 27.9$ & 0.01 & $7.9 \times 10^{8}$ \\
$3.55 / 0.54(\mathrm{sdB})$ & 1.1 & $3.5 / 29.4$ & 0.04 & $1.0 \times 10^{8}$ \\
$3.55 / 0.53(\mathrm{sdB})$ & 4.0 & $3.5 / 6.9$ & 0.04 & $1.1 \times 10^{8}$ \\
$5.01 / 0.46(\mathrm{sdB})$ & 1.1 & $1.6 / 27.2$ & 0.03 & $1.7 \times 10^{8}$ \\
$5.01 / 0.61(\mathrm{sdOB})$ & 1.1 & $2.5 / 29.0$ & 0.05 & $0.7 \times 10^{8}$ \\
$5.01 / 0.91(\mathrm{sdO})$ & 1.1 & $15.7 / 100.5$ & 0.09 & $0.2 \times 10^{8}$ \\
$5.01 / 0.98(\mathrm{sdOB})$ & 1.1 & $41.6 / 483.2$ & 0.12 & $0.2 \times 10^{8}$ \\
$5.01 / 1.00(\mathrm{sdB})$ & 1.1 & $43.6 / 526.0$ & 0.14 & $0.1 \times 10^{8}$ \\
$5.01 / 0.84(\mathrm{sdO})$ & 4.0 & $5.1 / 9.1$ & 0.08 & $0.3 \times 10^{8}$ \\
$5.01 / 0.91(\mathrm{sdO})$ & 4.0 & $32.3 / 59.4$ & 0.09 & $0.2 \times 10^{8}$ \\
\hline
\end{tabular}

$M_{1 \mathrm{i}}=$ initial mass; $M_{\mathrm{f}}=$ the mass of hot subdwarfs; $q_{\mathrm{i}}=$ the mass ratio; $P_{\mathrm{i}}=$ initial orbital period; $P_{\mathrm{f}}=$ orbital period of hot subdwarf binaries; $M_{\text {env }}=$ the envelope mass of hot subdwarfs; $t_{\mathrm{age}}=$ the lifetime of a hot subdwarf. More parameters can be seen from Figs. 2, 3, and 6-9.

different stages affects not only the surface gravity of hot subdwarfs but also their effective temperature given $q_{\mathrm{i}}=1.1$. The surface gravity decreases with the increase of the initial orbital period. For $q_{\mathrm{i}}=4.0$, the onset of mass transfer at different stages almost does not affect the effective temperature of hot subdwarfs, but the surface gravity decreases slightly with the initial orbital period.

In the progenitors of hot subdwarfs, the possibility of the onset of mass transfer at the main sequence decreases with mass ratio $q_{\mathrm{i}}$. In order to understand the mass transfer at different stages 


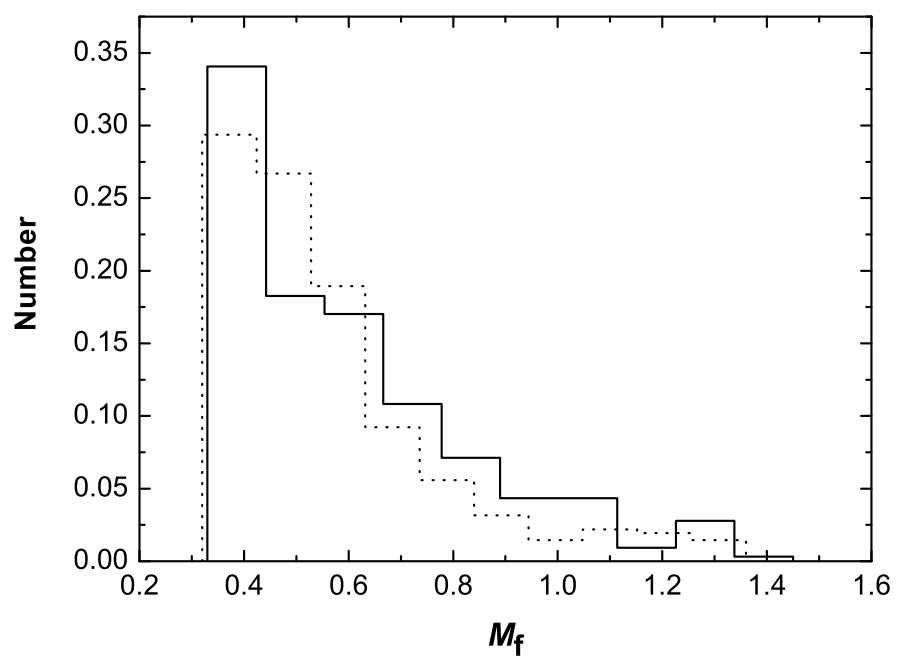

Fig. 11. The distributions of the masses of hot subdwarfs from the stable RLOF channel in this paper. $M_{\mathrm{f}}$ is the mass of hot subdwarfs. The solid and dotted line indicate the conservative and nonconservative cases, respectively.

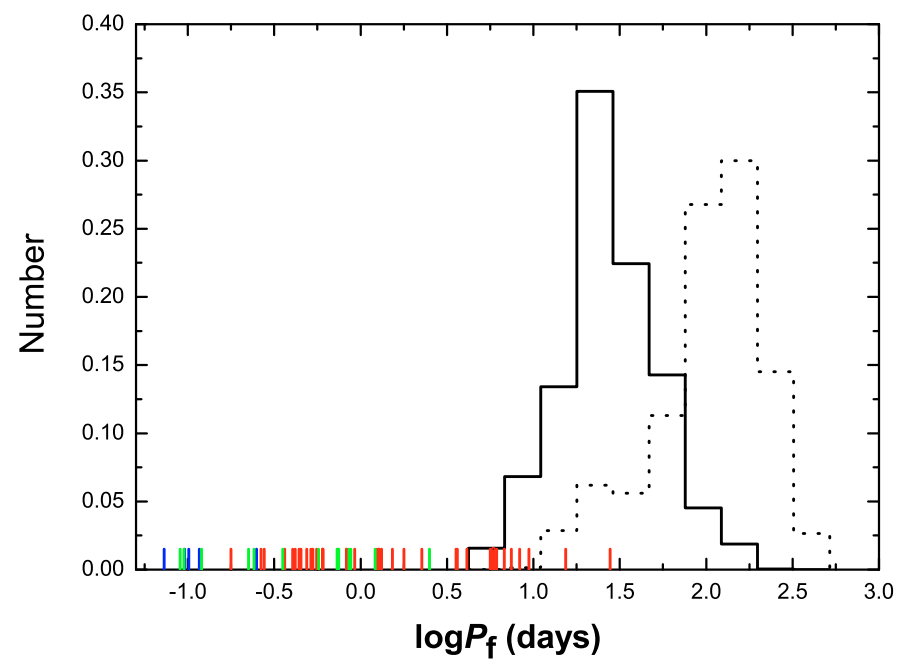

Fig. 12. Orbital-period distributions for stable RLOF hot subdwarfs. The solid and dotted line indicate the conservative and nonconservative cases, respectively. The red, blue and green bar along the period axis indicate the orbital periods of observed binaries with a companion of unknown type, main sequence and compact object, respectively (Morales-Rueda et al. 2003; Edelmann et al. 2005; Napiwotzki et al. 2004).

of the primary with different initial mass, we have plotted integral evolutionary tracks of the primary with different mass ratios in the HR diagram and mass transfer rate in Fig. 14. From Fig. 14, we see that the earlier the mass transfer begins, the lower the mass transfer rate. However, mass transfer beginning at an early stage tends to leave an sdB star. With an increase of initial orbital period, mass transfer rates increase steadily, even up to $10^{-4} M_{\odot} \mathrm{yr}^{-1}$, but the timescale of mass transfer of $10^{5} \mathrm{yr}$ is quite short. Some binaries with high initial mass and long initial orbital period would be on the brink of experiencing a delayed dynamical instability.

\subsection{Birth rate and number of hot subdwarfs from the stable RLOF channel}

Table 4 lists the birth rates of hot subdwarfs formed in various formation channels. In the table, the first column denotes
Table 4. Birth rates of hot subdwarfs from the stable RLOF channel of intermediate-mass binaries (our model) and other channels (Han et al. 2003) (in $10^{-3} \mathrm{yr}^{-1}$ ).

\begin{tabular}{lccccccc}
\hline \hline$Z$ & $q_{\text {crit }}$ & $v_{\mathrm{IMR}}$ & $v_{\mathrm{FR}}$ & $v_{\mathrm{FCE}}$ & $v_{\mathrm{SR}}$ & $v_{\mathrm{SCE}}$ & $v_{\mathrm{M}}$ \\
\hline 0.02 & 1.5 & 4.18 & 29.89 & 8.41 & 0.00 & 8.38 & 17.22 \\
0.02 & 1.2 & 4.14 & 22.25 & 10.71 & 0.00 & 5.43 & 7.99 \\
\hline
\end{tabular}

$Z=$ metallicity; $q_{\text {crit }}=$ the critical mass ratio for the first stable RLOF on the FGB; $v_{\mathrm{IMR}}=$ Galactic birth rates of hot subdwarfs from the stable RLOF channel of intermediate-mass binaries; $v_{\mathrm{FR}}=$ the first stable RLOF channel; $v_{\mathrm{FCE}}=$ the first CE ejection channel; $v_{\mathrm{SR}}=$ the second RLOF channel; $v_{\mathrm{SCE}}=$ the second $\mathrm{CE}$ ejection channel; $v_{\mathrm{M}}=$ the helium WD merger channel.

the metallicity ( $Z=0.02$ for Population I); the second column gives $q_{\text {crit }}$, the critical mass ratio for the first stable RLOF on the FGB; Cols. 3-8 list Galactic birth rates for hot subdwarfs (in $10^{-3} \mathrm{yr}^{-1}$ ) from the stable RLOF channel of intermediate-mass binaries (our model), the first stable RLOF channel, the first CE ejection channel, the second RLOF channel, the second CE ejection channel and the helium WD merger channel.

As Table 4 shows, the predicted birth rate of Population I hot subdwarfs from the stable RLOF channel of intermediatemass binaries is $0.004 \mathrm{yr}^{-1}$ for the Galaxy. By taking an effective Galactic volume of $5 \times 10^{11} \mathrm{pc}^{3}$ (Zombeck 1990), we give an average birth rate per $\mathrm{pc}^{3}$ of $8 \times 10^{-15} \mathrm{pc}^{-3} \mathrm{yr}^{-1}$. When convolved with the lifetime of hot subdwarf phase, this rate implies a total number of hot subdwarfs from the stable RLOF channel of intermediate-mass binaries in the Galaxy of $0.8 \times 10^{6}$. Comparing this result with the result of Han et al. (2003), we conclude that the stable RLOF channel of intermediate-mass binaries is important.

In order to compare our results with observed hot subdwarfs in the $T_{\text {eff- }} \log (g)$ diagram, we plot a grey diagram as Fig. 15. Comparing this figure with Fig. 4, we suppose that some hot subdwarfs with $T_{\text {eff }}>30000 \mathrm{~K}$ and $\log (g)>6.0$ might be evolved sdB stars.

\subsection{The effect of the secondary}

Most hot subdwarfs contain a main sequence (MS) or a subgiant star in our models. We discuss the effect of the secondary on observations for hot subdwarfs with a companion MS star in this section.

The luminosity of the secondary $(Z=0.02)$ is fitted by Tout et al. (1996) as:

$$
L=\frac{\alpha M^{5.5}+\beta M^{11}}{\gamma+M^{3}+\delta M^{5}+\varepsilon M^{7}+\zeta M^{8}+\eta M^{9.5}},
$$

where $M$ is the mass of a main sequence star, and $\alpha, \beta, \gamma, \delta, \varepsilon, \zeta, \eta$ are constants (see Tout et al. 1996). Our calculation indicates that if $q_{\mathrm{i}} \leq 2.0$, the luminosity of almost all hot subdwarfs is lower than that of their companions. This implies that these hot subdwarfs would not be detected by ground-based telescopes, although they could be found by space telescopes. If $q_{\mathrm{i}} \geq 3.0$, the luminosity of most hot subdwarfs will be larger than that of their companions, and this indicates that these hot subdwarfs might be observed by ground-based telescope. So, we suggest that a small fraction of observed hot subdwarfs could have progenitors with $q_{\mathrm{i}}$ larger than $\sim 3.0$. 

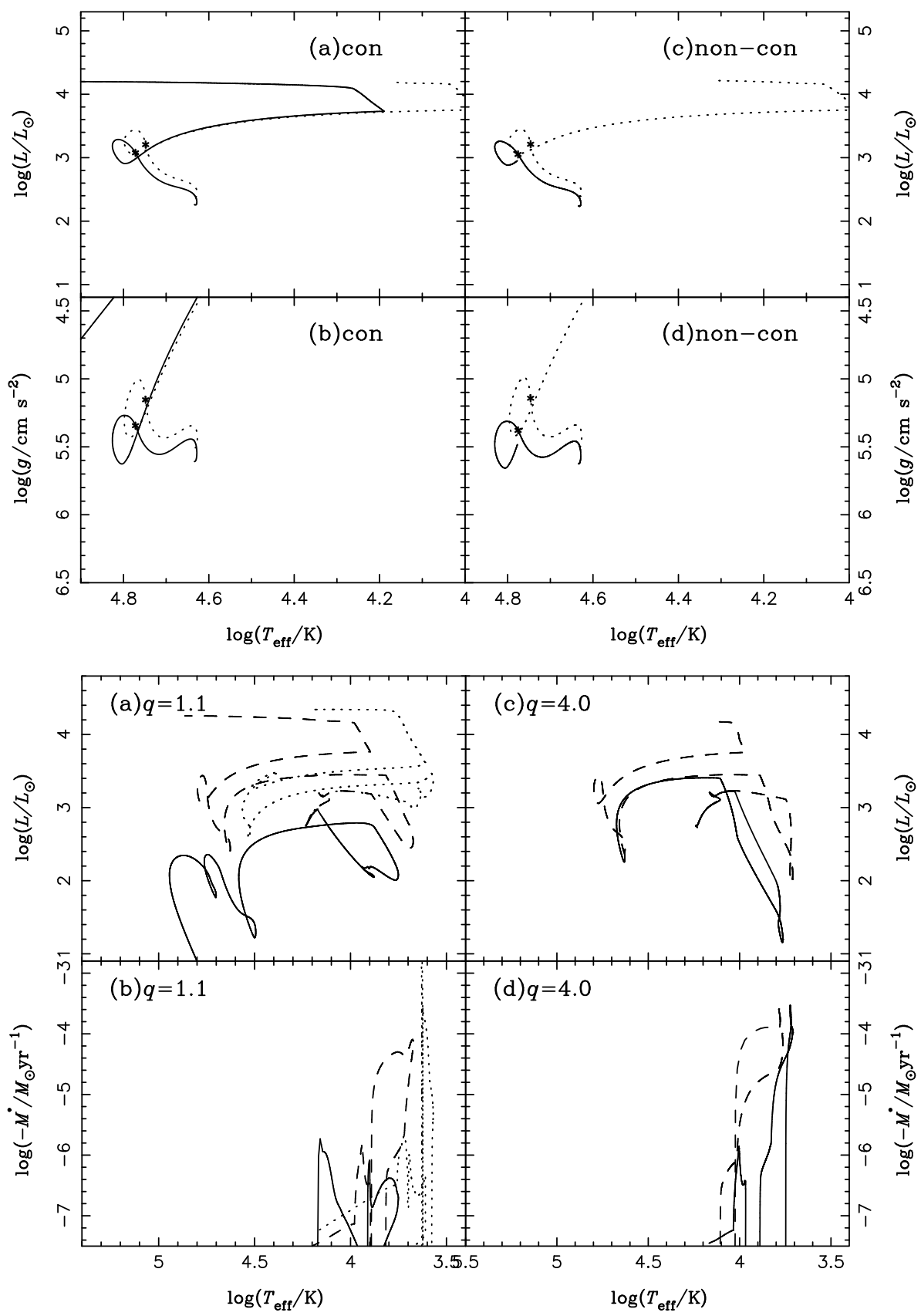

Fig. 13. The HR diagrams and the $T_{\text {eff }}-\log g$ diagrams of hot subdwarfs with progenitor's initial mass $M_{1 \mathrm{i}}$ of $5.01 M_{\odot}$ and initial mass ratio $q_{\mathrm{i}}$ is 4.0. All solid curves are for initial orbital periods of 5.1 days (sdO stars) and all dot-dashed for 32.3 days (sdO stars). Panels a) and b) are for the conservative case. Panels c) and d) are for the non-conservative case. Asterisks indicate that helium is exhausted in its center.

Fig. 14. Integral evolutionary tracks (top panels) and mass transfer rate (bottom panels) of the primary with initial mass of $M_{1 \mathrm{i}}=5.01 M_{\odot}$ in the non-conservative case. The left panels are for $q=1.1$, and the right panels are for $q=4.0$. In the left panels, solid curves are for the initial orbital periods of 1.6 days, mass transfer beginning at the MS stage (sdB star, with envelope mass of $0.03 M_{\odot}$ ), dashed for 15.7 days, mass transfer beginning at the HG stage (sdO star with envelope mass of $0.09 M_{\odot}$ ) and dot-dashed for 43.6 days, mass transfer beginning at the FGB stage (sdB star with an envelope mass of $0.14 M_{\odot}$ ). In the right panels, solid curves are for the initial orbital periods of 5.1 days, mass transfer beginning at the HG stage (sdO star with an envelope mass of $0.08 M_{\odot}$ ) and dashed curves for 32.3 days, mass transfer beginning at the HG stage (sdO star with an envelope mass of $0.09 M_{\odot}$ ).

\section{Discussion}

Han et al. (2002) have proposed a binary model for the formation of hot subdwarfs, including three channels: the CE ejection channel, the stable RLOF channel and the double helium WD merger channel. In the $\mathrm{CE}$ ejection channel, a primary experiences dynamically unstable mass transfer at the tip of the FGB and leads to the formation of a CE (Paczyński 1976). During the spiral-in process in the CE, the orbit of the binary shrinks due to the friction between binary and envelope. The CE will be ejected if the released orbital energy exceeds the binding energy in this process, and this process would leave a very tight binary containing the degenerate core of the giant. If the degenerate core experiences a helium flash, the remnant core of the giant may be ignited (Castellani \& Castellani 1993) and hence appear as a core-helium-burning hot subdwarf in binaries with short orbital periods in the range of 0.05-40 days (Han et al. 2002). In stable RLOF channel, a primary undergoes a stable mass transfer at the FGB stage. If its helium core was ignited after the RLOF, the primary would become a hot subdwarf in binaries with long orbital periods in the range of 400-1500 days (Han et al. 2002). Our results indicate that the mass transfer can also begin at the MS stage (case A mass transfer) and HG stage (case B mass transfer). This leads to hot subdwarfs in binaries with a wide orbital period range of 5-900 days. Compared with the CE ejection channel, we found that there are a large number of hot subdwarfs in binaries with long orbital periods formed through the stable RLOF channel. This would sustain the observations by Green et al. (2000) and Morales-Rueda et al. (2003), who argued that some sdB stars could appear to be members of long period binaries. Moreover, it is possible that some hot subdwarfs in binaries with a very long orbital period could be misunderstood as single stars.

In general, sdB stars are believed to have a mass of around $0.5 M_{\odot}$ and a thin hydrogen rich envelope around $0.02 M_{\odot}$ (Heber 1986; Saffer et al. 1994). Han et al. (2002) suggested that the mass of sdB stars is in the range of $0.33-0.68 M_{\odot}$. In 


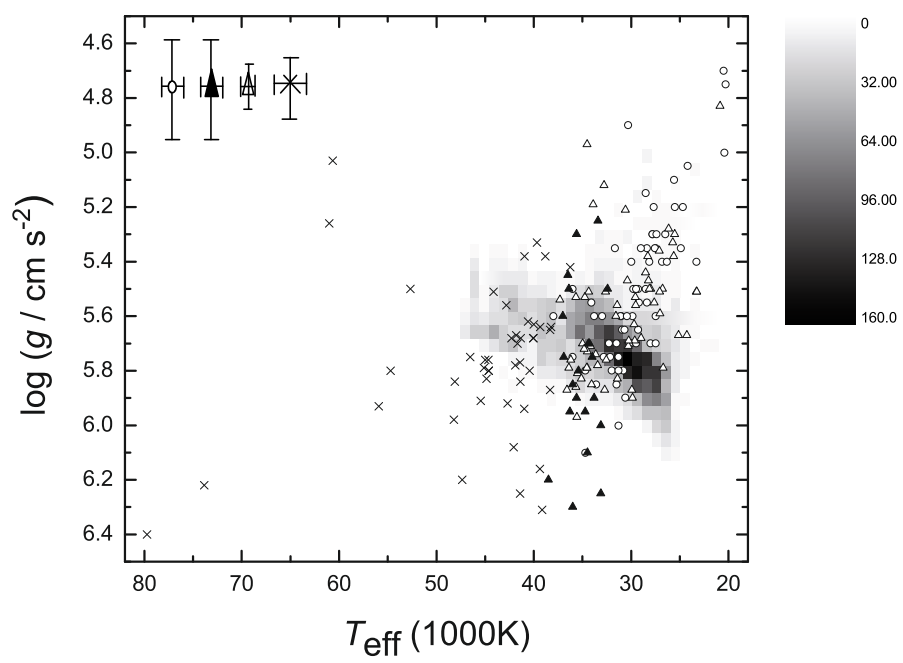

Fig. 15. Comparison of our results to the atmospheric parameters of sdB stars (open circles Edelmann et al. 2003; open triangles Lisker et al. 2005), sdOB stars (solid triangles Edelmann et al. 2003), sdO stars (crosses Stroeer et al. 2007). The largest cross is for HE 1115-0631 (orbital period: $5.87 \mathrm{~d}$, Napiwotzki et al. 2004). Our results are shown as shaded $T_{\text {eff }}-\log (g)$-boxes, where a higher hot subdwarf density per box corresponds to darker shading. The grey scale is shown in the right of the figure.

our models, the mass of most sdB stars is in the range of $0.32-$ $0.67 M_{\odot}$ with a thin envelope of $0.01-0.05 M_{\odot}$, but a few sdB stars have an anomalously high mass of $0.72-1.13 M_{\odot}$, and a thick envelope of $0.07-0.16 M_{\odot}$. The reasons have been described in Sect. 3. The mass of sdO stars is from $0.75 M_{\odot}$ to $1.44 M_{\odot}$ and its envelope mass is higher than $0.07 M_{\odot}$, even up to $0.22 M_{\odot}$. The mass and the envelope mass of sdOB stars are between sdB stars and sdO stars. In addition, Han et al. (2000) and Chen et al. (2002) discussed low- and intermediate-mass close binary evolution and the initial-final mass relation of these binaries. By calculating a binary evolution sequence with an initial mass ratio between 1.1 and 4.0, and the onset of the stable RLOF at the Hertzsprung gap stage, they found that the remnant mass of the primary depends on the initial mass ratio or orbital period for a given primary mass. These results are similar to ours, but binary evolution calculations with the onset of mass transfer at the main sequence stage have been included in our models.

Elson et al. (1998) discovered a blue star with $T_{\text {eff }} \approx$ $31500 \mathrm{~K}, \log (g) \approx 4.4, \log L / L_{\odot} \sim 3.0$ (Burleigh et al. 1999) in the young cluster NGC 1818 in the Large Magellanic Cloud (LMC), which has a main-sequence turnoff mass of $\sim 7.5-$ $9.5 M_{\odot}$, an age of $\sim 2-4 \times 10^{7} \mathrm{yr}$ (Will et al. 1995) and a metallicity of $\sim 0.005$ (Kerber \& Santiago 2005). This star was considered as a candidate of luminous WD. However, Liebert (1999) and Burleigh et al. (1999) pointed out that the star would be a post-EHB star or EHB star, rather than a WD, and it would lie in the Galactic halo or the disc of the LMC instead of cluster NGC 1818. If this blue star was in a binary system, we suggest that the star could be in the cluster NGC 1818 and form through the stable RLOF channel of intermediate-mass binaries, but this needs more observational and theoretical evidence to confirm. We consider that hot subdwarfs could exist in young clusters, and some of them might have high mass $\left(>0.7 M_{\odot}\right)$. We will focus on hot subdwarfs in young clusters and calculate a binary evolution sequence with different metallicity $(Z)$ to study their properties, formation and evolution in the future.
Mass transfer efficiency is an important factor affecting the initial parameter spaces, as lower mass transfer efficiency will lead to more binaries experiencing stable RLOF rather than the formation of a common envelope during rapid mass transfer. Past work (Paczyński \& Ziółkowski 1967; Refsdal et al. 1974; De Greve \& De Loore 1992; Chen \& Han 2002, 2003) employed mass transfer efficiency $\beta=0.5$ for the calculation of stellar evolution in nonconservative cases. However, De Mink et al. (2007) suggested that mass transfer efficiency should not be a single constant by comparing their models with a sample of 50 doublelined eclipsing binaries in the Small Magellanic Cloud, which could be affected by spin up of the accreting star and tidal interaction. They also found that initially wider systems tend to favor less conservative models, since accreting angular momentum will speed up the rotation of the accreting star, resulting in mass loss along its equator region, as discussed by Wellstein (2001) and Petrovic et al. (2005). Our results are in line with this conclusion. Due to the uncertain of the mass transfer efficiency (De Mink et al. 2007), the birth rate and the total number from our models need further data to constrain. However, it is reasonable to estimate that a high birth rate and large total number of hot subdwarfs via stable RLOF channel will suggest less conservative models.

Other effects on the birth rate and total number of hot subdwarfs in our models would arise from the life-time of the secondary, which will become too short, such that it is comparable to or even smaller than the lifetime of the hot subdwarf phase (typical value: $2.0 \times 10^{8} \mathrm{yr}$ ) in some extreme cases. In these two cases, the secondary presumably evolved rapidly to the giant branch or even to later stages after fast mass transfer, while the primary could be a giant or a hot subdwarf or a helium star, which could be associated with a "post-Algol" binary (Nelson \& Eggleton 2001). As a result, we could overestimate the birth rate and the total number of hot subdwarfs via the stable RLOF channel.

\section{Conclusions}

We present the properties of hot subdwarfs from the stable RLOF channel of intermediate-mass binaries by computing population I binary evolution sequences both in conservative and nonconservative cases, where the primary has an initial mass in the range of $2.2-6.3 M_{\odot}$, initial mass ratio $q_{\mathrm{i}}=1.1,1.5,2.0$, 3.0, 4.0, 4.2, 4.5, and onset of RLOF at the MS, HG, and FGB stage. Due to the effect of the secondary, the ratio of the hot subdwarf bianries which might be observed by ground-based telescope would be larger around 3.0. We summarize our results as follows:

1. The birth rate of hot subdwarfs from the stable RLOF channel of intermediate-mass binaries is $\sim 4.2 \times 10^{-3} \mathrm{yr}^{-1}$, which is similar to the first $\mathrm{CE}$ ejection channel. However, the current observations indicate that only a few hot subdwarfs binaries could be formed via the stable RLOF channel (Fig. 12). This could be associated with the rotation of the accreting star and the evolution of the secondary.

2. We have obtained the initial parameter space of the progenitor binaries of hot subdwarfs from the stable RLOF channel of intermediate-mass binaries (Figs. 2 and 3).

3. Our results indicate that the hot subdwarfs from the stable RLOF channel of intermediate-mass binaries have a mass in the range of $0.32-1.44 M_{\odot}$ with a wide envelope mass of 0.01-0.22 $M_{\odot}$. Most sdB stars have a mass in the range of $0.32-0.67 M_{\odot}$ with an envelope mass of $0.01-0.05 M_{\odot}$. 
Most sdOB stars have a mass in the range of $0.60-0.86 M_{\odot}$ with an envelope mass of $0.05-0.10 M_{\odot}$. SdO stars have a mass in the range of $0.75-1.44 M_{\odot}$ with an envelope mass of $0.07-0.22 M_{\odot}$. Furthermore, we found that some sdB or sdOB stars have anomalously high mass, in the range of $0.72-1.13 M_{\odot}$ and $0.97-1.23 M_{\odot}$, respectively. The envelope mass is in the range of $0.07-0.16 M_{\odot}$ and $0.11-0.19 M_{\odot}$, respectively. We expect that observations could confirm these theoretical results in the future.

4. The orbital periods of the binaries have a wide range of 5.0900.0 days, while the peak is at around 120 days for the conservative case and 30 days for the nonconservative case.

5. Our results favor that hot subdwarfs, formed through the RLOF channel of intermediate-mass binaries, will be found in young clusters in the future.

Acknowledgements. We would like to acknowledge Zhanwen Han, Fenghui Zhang and Xuefei Chen for their discussions and suggestions and also thank the referee and editor for their useful suggestions and comments.

\section{References}

Alexander, D. R., \& Ferguson, J. W. 1994a, in Molecules in the Stellar Environment, ed. U. G. Jorgensen (Berlin: Springer-Verlag), 149

Alexander, D. R., \& Ferguson, J. W. 1994b, ApJ, 437, 879

Andersen, J. 1991, A\&ARv, 3, 91

Baschek, B., \& Norris, J. 1975, ApJ, 199, 694

Berger, J. 1963, Pub. ASP, 75, 393

Bessell, M. S., Brett, J. M., Scholz, M., \& Wood, P. R. 1989, A\&AS, 77, 1

Burleigh, M. R., Saffer, R. A., Gilmore, G. F., et al. 1999, MNRAS, 310, L1

Carraro, G., Girardi, L., Bressan, A., \& Chiosi, C. 1996, A\&A, 305, 849

Castellani, M., \& Castellani, V. 1993, ApJ, 407, 649

Caughlan, G. R., \& Fowle, W. A. 1988, At. Data Nucl. Data Tables, 40, 284

Caughlan, G. R., Fowler, W. A., Harris, M. J., \& Zimmerman, B. A. 1985, At. Data Nucl. Data Tables, 35, 198

Chen, X. F., \& Han, Z. 2002, MNRAS, 335, 948

Chen, X. F., \& Han, Z. 2003, MNRAS, 341, 662

D'Cruz, N. L., Dorman, B., Rood, R. T., \& O'Connell, R. W. 1996, ApJ, 466, 359

De Greve, J. P., \& De Loore, C. 1992, A\&AS, 96, 653

De Mink, S. E., Pols, O. R., \& Hilditch, R. W. 2007, A\&A, 467, 1181

Dorman, B., Rood, R. T., \& O'Connell, R. W. 1993, ApJ, 419, 596

Edelmann, H., Heber, U., Hagen, H.-J., et al. 2003, A\&A, 400, 939

Edelmann, H., Heber, U., Altmann, M., et al. 2005, A\&A, 442, 1023

Elson, R. A., Sigurdsson, S., Hurley, J., et al. 1998, ApJ, 499, L53

Eggen, O. J. 1985, AJ, 90, 333

Eggleton, P. P. 1971, MNRAS, 151, 351

Eggleton, P. P. 1972, MNRAS, 156, 361

Eggleton, P. P. 1973, MNRAS, 163, 179

Eggleton, P. P., Fitchett, M. J., \& Tout, C. A. 1989, ApJ, 347, 998

Feige, J. 1958, ApJ, 128, 267

Goldberg, D., \& Mazeh, T. 1994, A\&A, 282, 801

Green, E. M., Liebert, J., \& Saffer, R. A. 2000, [arXiv: astro-ph/0012246]

Greenstein, J. L. 1966, ApJ, 144, 496

Han, Z. 1998, MNRAS, 296, 1019

Han, Z., Podsiadlowski, Ph., \& Eggleton, P. P. 1994, MNRAS, 270, 121

Han, Z., Eggleton, P. P., Podsiadlowski, Ph., \& Tout, C. A. 1995, MNRAS, 277, 1443

Han, Z., Tout, C. A., \& Eggleton, P. P. 2000, MNRAS, 319, 215

Han, Z., Podsiadlowski, Ph., Maxted, P. F. L., Marsh, T. R., \& Ivanova, N. 2002, MNRAS, 336, 449
Han, Z., Podsiadlowski, Ph., Maxted, P. F. L., \& Marsh, T. R. 2003, MNRAS, 341, 669

Han, Z., Podsiadlowski, Ph., Lynas-Gray, A. E., \& Schawinski, K. 2007, IAUS, 241,181

Heber, U. 1986, A\&A, 155, 33

Humason, M. S., \& Zwicky, F. 1947, ApJ, 105, 85

Hunger, K., Gruschinske, J., Kudritzki, R. P., \& Simon, K.P. 1981, A\&A, 95, 244

Iben, I., Jr, \& Renzini, A. 1983, ARA\&A, 21, 271

Iben, I., Jr, \& Tutukov, A. V. 1986, ApJ, 311, 753

Iglesias, C. A., \& Rogers, F. G. 1996, ApJ, 464, 943

Itoh, N., Mutoh, H., Hikita, A., Kohyama, Y., \& Munakata, H. 1989, ApJ, 339, 354 (erratum 1990, ApJ, 360, 741)

Itoh, N., Mutoh, H., Hikita, A., \& Kohyama, Y. 1992, ApJ, 395, 622 (erratum 1993, ApJ, 404, 418)

Kerber, L. O., \& Santiago, B. X. 2005, A\&A, 435, 77

Klemola, A. R. 1962, AJ, 67, 40

Kroupa, P., Tout, C. A., \& Gilmore, G. 1993, MNRAS, 262, 545

Lee, Y. W. 1994, ApJ, 430, L113

Liebert, J. 1999, ApJ, 514, L25

Lisker, T., Heber, U., Napiwotzki, R., et al. 2005, A\&A, 430, 223

Maxted, P. F. L., Heber, U., Marsh, T. R., \& North, R. C. 2001, MNRAS, 326, 1391

Mazeh, T., Goldberg, D., Duquennoy, A., \& Mayor, M. 1992, ApJ, 401, 265

Miller, G. E., \& Scalo, J. M. 1979, ApJS, 41, 513

Moni Bidin, C., Moehler, S., Piottol, G., et al. 2006, A\&A, 451, 499

Moni Bidin, C., Moehler, S., Piotto, G., et al. 2006, [arXiv: astro-ph/0606035v1]

Morales-Rueda, L., Maxted, P. F. L., Marsh, T. R., et al. 2003, MNRAS, 338, 752

Morales-Rueda, L., Maxted, P. F. L., Marsh, T. R., et al. 2005, ASPC, 334, 333

Napiwotzki, R., Karl, C. A., Lisker, T., et al. 2004, Ap\&SS, 291, 321

Nelson, C. A., \& Eggleton, P. P. 2001, ApJ, 552, 664

Paczyński, B. 1971, Acta Astron., 21, 1

Paczyński, B. 1976, in Structure and Evolution of Close Binaries, ed. P. P. Eggleton, S. Mitton, J. Whelan (Dordrecht: Kluwer), 75

Paczyński, B., \& Ziółkowski, J. 1967, Acta Astron., 17, 7

Park, J. H., \& Lee, Y. W. 1997, ApJ, 476, 28

Petrovic, J., Langer, N., Yoon, S.-C., \& Heger, A. 2005, A\&A, 435, 247

Podsiadlowski, Ph., Rappaport, S., \& Pfahl, E. D. 2002, ApJ, 565, 1107

Pols, O. R., Tout, C. A., Eggleton, P. P., \& Han, Z. 1995, MNRAS, 274, 964

Pols, O. R., Tout, C. A., Schröder, K.-P., Eggleton, P. P., \& Manners, J. 1997, MNRAS, 289, 869

Pols, O. R., Schröder, K.-P., Hurley, J. R., Tout, C. A., \& Eggleton, P. P. 1998, MNRAS, 298, 525

Refsdal, S., Roth, M. L., \& Weigert, A. 1974, A\&A, 36, 113

Reimers, D. 1975, Mem. R. Soc. Liége, 6e Ser., 8, 369

Renzini, A. 1981, in Effects of Mass Loss on Stellar Evolution, ed. C. Chiosi, R. Stalio (Dordrecht: Reidel), 319

Saffer, R. A., Bergeron, P., Koester, D., \& Liebert, J. 1994, ApJ, 432, 351

Sargent, W. L. W., \& Searle, L. 1968, ApJ, 152, 443

Schröder, K.-P., Pols, O. R., \& Eggleton, P. P. 1997, MNRAS, 285, 696

Slettebak, A., Bahner, K., \& stock, J. 1961, ApJ, 134, 195

Stroeer, A., Heber, U., Lisker, T., et al. 2007, A\&A, 462, 269

Sweigart, A. V. 1997, ApJ, 474, L23

Tout, C. A., Pols, O. R., Eggleton, P. P., et al., 1996, MNRAS, 281, 257

Webbink, R. F. 1984, ApJ, 277, 355

Wellstein, S. 2001, Ph.D. Thesis

Will, Jean-Marie, Bomans, Dominik J., \& de Boer Klaas, S. 1995, A\&A, 295, 54

Yi, S. K., \& Yoon, Suk-Jin 2004, Ap\&SS, 291, 205

Yi, S., Demarque, P., \& Oemler, A., Jr 1997, ApJ, 486, 201

Zoccali, M., Cassisi, S., Frogel, J. A., et al. 2000, ApJ, 530, 418

Zombeck, M. V. 1990, Handbook of Astronomy and Astrophysics, 2nd edn. (Cambridge: Cambridge Univ. Press) 